\title{
Functional signatures of oral dysbiosis during periodontitis progression revealed by microbial metatranscriptome analysis
}

\author{
Susan Yost $^{1 \dagger}$, Ana E Duran-Pinedo ${ }^{1 \dagger}$, Ricardo Teles ${ }^{3}$, Keerthana Krishnan ${ }^{1}$ and Jorge Frias-Lopez ${ }^{1,2^{*}}$
}

\begin{abstract}
Background: Periodontitis is a polymicrobial biofilm-induced inflammatory disease that affects 743 million people worldwide. The current model to explain periodontitis progression proposes that changes in the relative abundance of members of the oral microbiome lead to dysbiosis in the host-microbiome crosstalk and then to inflammation and bone loss. Using combined metagenome/metatranscriptome analysis of the subgingival microbiome in progressing and non-progressing sites, we have characterized the distinct molecular signatures of periodontitis progression.

Methods: Metatranscriptome analysis was conducted on samples from subgingival biofilms from progressing and stable sites from periodontitis patients. Community-wide expression profiles were obtained using Next Generation Sequencing (Illumina). Sequences were aligned using 'bowtie2' against a constructed oral microbiome database. Differential expression analysis was performed using the non-parametric algorithm implemented on the R package 'NOISeqBio'. We summarized global functional activities of the oral microbial community by set enrichment analysis based on the Gene Ontology (GO) orthology.

Results: Gene ontology enrichment analysis showed an over-representation in the baseline of active sites of terms related to cell motility, lipid A and peptidoglycan biosynthesis, and transport of iron, potassium, and amino acids. Periodontal pathogens (Tannerella forsythia and Porphyromonas gingivalis) upregulated different TonB-dependent receptors, peptidases, proteases, aerotolerance genes, iron transport genes, hemolysins, and CRISPR-associated genes. Surprisingly, organisms that have not been usually associated with the disease (Streptococcus oralis, Streptococcus mutans, Streptococcus intermedius, Streptococcus mitis, Veillonella parvula, and Pseudomonas fluorenscens) were highly active transcribing putative virulence factors. We detected patterns of activities associated with progression of clinical traits. Among those we found that the profiles of expression of cobalamin biosynthesis, proteolysis, and potassium transport were associated with the evolution towards disease.
\end{abstract}

Conclusions: We identified metabolic changes in the microbial community associated with the initial stages of dysbiosis. Regardless of the overall composition of the community, certain metabolic signatures are consistent with disease progression. Our results suggest that the whole community, and not just a handful of oral pathogens, is responsible for an increase in virulence that leads to progression.

Trial registration: NCT01489839, 6 December 2011.

\footnotetext{
* Correspondence: jfrias@forsyth.org

†'Equal contributors

${ }^{1}$ Forsyth Institute, 245 First Street, Cambridge, Massachusetts 02142, USA

${ }^{2}$ Harvard School of Dental Medicine, 188 Longwood Ave, Boston, MA

02115, USA

Full list of author information is available at the end of the article
}

() Biomed Central

(c) 2015 Yost et al.; licensee BioMed Central. This is an Open Access article distributed under the terms of the Creative Commons Attribution License (http://creativecommons.org/licenses/by/4.0), which permits unrestricted use, distribution, and reproduction in any medium, provided the original work is properly credited. The Creative Commons Public Domain Dedication waiver (http://creativecommons.org/publicdomain/zero/1.0/) applies to the data made available in this article unless otherwise stated. 


\section{Background}

Much has been learned about the diversity and distribution of oral associated microbial communities, but we still know little about the biology of the microbiome, how it interacts with the host, and how the host responds to its resident microbiota. The oral cavity offers a unique opportunity to study how microbial communities have an influence on the health status of their human host. Imbalances of the oral microbiota, also referred to as microbial dysbiosis, lead to a series of different oral diseases. These dysbiotic microbial communities exhibit synergistic interactions for enhanced protection from host defenses, nutrient acquisition, and persistence in an inflammatory environment [1,2]. Periodontitis is an oral polymicrobial disease caused by the coordinated action of a complex microbial community, which results in inflammation and destruction of the periodontium in susceptible hosts. Periodontal disease is the sixth most prevalent health condition in the world affecting 743 million people worldwide [3]. It occurs in moderate form in $30 \%$ to $50 \%$ of American adults and in severe form in $10 \%$ of the population and is responsible for half of all tooth loss in adults $[4,5]$. In addition, recent studies have suggested that periodontal diseases can influence the risk for certain systemic conditions such as cardiovascular diseases, diabetes, respiratory diseases, and can affect reproductive outcomes [6,7].

Using checkerboard, a DNA-DNA hybridization technique, periodontitis-associated taxa have been cataloged into groups or complexes, representing bacterial consortia that appear to occur together and that are associated with various stages of disease [7]. The red complex, which appears later in biofilm development, comprises three species that are considered to be the major periodontal pathogens: Porphyromonas gingivalis, Treponema denticola, and Tannerella forsythia [7,8]. Another important group of organisms that has been associated with chronic periodontitis is the orange complex constituted by: Fusobacterium nucleatum, Prevotella intermedia, Prevotella nigrescens, Parvimonas micra, Streptococcus constellatus, Eubacterium nodatum, Campylobacter showae, Campylobacter gracilis, and Campylobacter rectus. Similar to the red complex, all species in the orange complex have been shown to have a significant association with increasing pocket depth $[7,9]$ and reciprocal interactions between both have been proposed [9]. In more recent studies using 454 pyrosequencing to characterize healthy and periodontitis microbial communities $[10,11]$, the overall picture of bacterial associations with health and disease agree with the initial descriptions of the different oral microbial complexes.

One key question to be answered regarding the pathogenesis of polymicrobial diseases is what the molecular mechanisms that lead to dysbiosis are. In the case of periodontitis, why in some cases teeth with clinical symptoms of periodontitis progress leading to tooth loss (if untreated) and in some other cases the progression of the disease stops despite lack of treatment [12]. There have been a large number of attempts to identify reliable markers that would distinguish between progressing and non-progressing sites. These include genetic markers $[13,14]$, protein activity $[15,16]$, cytokines $[17,18]$, and bacterial $[19,20]$ and clinical markers [19]. However, none of these associations explain why progression occurs.

Current models of periodontal disease progression posit that tissue destruction occurs through periods of acute exacerbations (activity) followed by periods of remission $[21,22]$. It has been postulated that changes in the composition of subgingival biofilms could explain these periods of disease activity. In fact, a few papers have found differences in the levels of subgingival species when comparing progressing and non-progressing sites using cultivation techniques [23] and molecular approaches such as real-time PCR [20]. However, these studies also demonstrated considerable overlap in the composition of the microbial communities associated with active and non-progressing lesions, suggesting that the difference in the periodontal status of the sites could not be explained solely by the reported differences in the subgingival microbial composition.

Cataloging the activities of each bacterial species in a community may provide more insight into pathogenesis than simple enumeration of that community's gene content. This is because the community functions as a system, and its activities and interactions control the fate of the microbiome.

The goal of the present study was to characterize in situ gene expression patterns of the whole oral microbiome during periodontitis progression to identify early steps in dysbiosis that could answer the question of why only certain teeth progress to disease while others do not.

\section{Methods}

\section{Ethics statement}

Written informed consent was obtained from all participants in this study. Recruitment of study participants was performed according to protocol (ClinicalTrials.gov ID NCT01489839) approved by Forsyth Institutional Review Board ID \#11-09 (Approval Date 8 August 2014) in Cambridge, MA, USA. All subjects provided written signed informed consent prior to participation.

\section{Power calculation}

In order to assess the sample size required we used the $R$ package RNASeqPower [24]. We first estimated the average coverage using samtools depth command from the samtools package [25]. According to this analysis, 
with an FDR of 0.05 and a target effect size of 2, we need eight subjects in each group to have a power of 0.9.

\section{Study design, subject population, and sample collection} The subjects in the present study were recruited as part of a multi-center clinical trial to determine biomarkers of periodontal disease progression (Clinical Trials.gov ID NCT01489839). Under this ongoing study, subjects are monitored clinically for a period of up to 1 year every 2 months in order to detect periodontal sites and subjects with periodontal disease progression. Subgingival microbial samples are collected from up to 32 sites per subject per visit. The Institutional Review Board at The Forsyth Institute approved all aspects of the study protocol. The study was described thoroughly to all subjects prior to obtaining informed consent. Inclusion criteria were: age $>24$ years; $\geq 20$ natural teeth (excluding third molars); at least four teeth with at least one site of pocket depth (PD) of $5 \mathrm{~mm}$ or more and concomitant clinical attachment loss (CAL) greater than or equal to $2 \mathrm{~mm}$; radiographic evidence of mesial or distal alveolar bone loss around at least two of the affected teeth; and in good general health (Table 1). Exclusion criteria were: current cigarette smokers; pregnant or nursing; received antibiotic or periodontal therapy in the previous 6 months; any systemic condition potentially affecting the course of periodontal disease (for example, diabetes or AIDS); chronic use of non-steroidal anti-inflammatory drugs; or any condition requiring antibiotic coverage for dental procedures. Disease progression at a site was defined by an increase in CAL $\geq 2 \mathrm{~mm}$ at any follow-up visit compared with baseline. Stable sites were characterized by no change in CAL $>1 \mathrm{~mm}$ from baseline. Eight stable sites and eight progressing sites from the nine subjects were analyzed (Table 2). We collected one stable site and one progressing site, both at baseline and at the endpoint of analysis. For seven of the nine subjects, both progressing and stable sites matched the initial baselines. Samples were processed as described below.
After removal of supragingival plaque, subgingival plaque samples were taken separately from the mesiobuccal and disto-buccal sites of pre-molars and first and second molars using individual sterile Gracey curettes and each sample placed in individual tubes containing $200 \mathrm{uL}$ of RNAse-free TE buffer, immediately frozen and stored at $-80^{\circ} \mathrm{C}$ until processed.

\section{Community DNA and RNA extraction}

Cells were collected by centrifugation for $10 \mathrm{~min}$ at maximum speed in a microcentrifuge. A total of $600 \mu \mathrm{L}$ of mirVana kit lysis/binding buffer and $300 \mu \mathrm{L}$ of 0.1$\mathrm{mm}$ zirconia-silica beads (BioSpec Products, Bartlesville, OK, USA) were added to the samples. The beads were treated with DEPC overnight and autoclaved. Samples were bead beaten for $1 \mathrm{~min}$ at maximum speed. DNA and RNA were extracted simultaneously following the protocol of mirVana ${ }^{\mathrm{TM}}$ Isolation kit for RNA and ToTALLY RNA ${ }^{\text {TM }}$ kit (Life Technologies) for DNA. Eukaryotic DNA was removed using the MolYsis ${ }^{\circ}$ kit (Molzym GmbH \& Co. KG, Bremen, Germany). MICROBioEnrich (Life Technologies) was used to remove eukaryotic RNA and MICROBExpress (Life Technologies) to remove prokaryotic rRNA. All kits were used following the manufacturers' instructions.

\section{DNA, RNA amplification, and Illumina sequencing}

DNA amplification was performed using the Illustra GenomiPhi V2 amplification kit (GE Healthcare Life Sciences) according to the manufacturer's instructions. RNA amplification was performed on total bacterial RNA using MessageAmp ${ }^{\text {TM }}$ II-Bacteria RNA amplification kit (Life Technologies) following the manufacturer's instructions. Sequencing was performed at the Forsyth Institute. Illumina adapter-specific primers were used to amplify and selectively enrich for the cDNA generated from enriched mRNA. Quantified libraries were pooled and sequenced using the MiSeq v2, $2 \times 150$ cycle cartridge (Illumina). The Nextera $\mathrm{XT}$ kit was used to

Table 1 Clinical and demographic characteristics of study subjects

\begin{tabular}{lllll}
\hline Subject & Age (years) & Mean PD $(\mathbf{m m})$ & Mean CAL $(\mathbf{m m})$ & Sites with PD $\geq \mathbf{5}$ mm (\%) \\
\hline 1 & 53 & 2.1 & 1.4 & $7 \%$ \\
2 & 32 & 2.5 & 1.8 & $15 \%$ \\
3 & 54 & 3.0 & 3.0 & $17 \%$ \\
4 & 3.9 & 3.3 & $41 \%$ & $21 \%$ \\
5 & 50 & 3.1 & 3.3 & $3 \%$ \\
6 & 59 & 2.1 & 2.5 & $10 \%$ \\
7 & 42 & 1.9 & 3.4 & $7 \%$ \\
8 & 75 & 2.0 & 2.2 & $4 \%$ \\
\hline
\end{tabular}

CAL: clinical attachment loss; PD: pocket depth. 
Table 2 Clinical characteristics of progressing and stable sites

\begin{tabular}{|c|c|c|c|c|c|}
\hline Subject & Site $^{a}$ & Visit (months) & $\mathrm{PD}(\mathrm{mm})$ & CAL $(\mathrm{mm})$ & Progression $(0 / 1)$ \\
\hline \multirow[t]{2}{*}{1} & 361 & 0 & 3.0 & 2.0 & 1 \\
\hline & 361 & 2 & 5.0 & 4.0 & \\
\hline \multirow[t]{2}{*}{1} & 353 & 0 & 2.5 & 1.5 & 0 \\
\hline & 353 & 2 & 3.0 & 2.0 & \\
\hline \multirow[t]{2}{*}{2} & 473 & 0 & 3.0 & 2.0 & 1 \\
\hline & 473 & 2 & 5.0 & 4.0 & \\
\hline \multirow[t]{2}{*}{2} & 241 & 0 & 3.0 & 1.0 & 0 \\
\hline & 241 & 2 & 3.0 & 2.0 & \\
\hline \multirow[t]{2}{*}{3} & 273 & 0 & 4.0 & 4.0 & 1 \\
\hline & 273 & 2 & 8.5 & 8.0 & \\
\hline \multirow[t]{2}{*}{3} & 441 & 0 & 4.0 & 3.0 & 0 \\
\hline & 441 & 2 & 3.0 & 2.0 & \\
\hline \multirow[t]{2}{*}{4} & 143 & 0 & 6.0 & 5.0 & 1 \\
\hline & 143 & 2 & 8.0 & 7.0 & \\
\hline \multirow[t]{2}{*}{4} & 453 & 0 & 4.5 & 3.5 & 0 \\
\hline & 453 & 2 & 5.0 & 4.0 & \\
\hline \multirow[t]{2}{*}{5} & 151 & 0 & 3.0 & 3.0 & 1 \\
\hline & 151 & 2 & 5.0 & 5.5 & \\
\hline \multirow[t]{2}{*}{5} & 373 & 0 & 3.0 & 3.0 & 0 \\
\hline & 373 & 2 & 3.0 & 3.0 & \\
\hline \multirow[t]{2}{*}{6} & 143 & 0 & 2.0 & 2.0 & 1 \\
\hline & 143 & 4 & 4.0 & 4.0 & \\
\hline \multirow[t]{2}{*}{6} & 151 & 0 & 1.5 & 2.5 & 0 \\
\hline & 151 & 2 & 1.0 & 2.0 & \\
\hline \multirow[t]{2}{*}{7} & 253 & 0 & 2.5 & 2.5 & 1 \\
\hline & 253 & 4 & 5.0 & 5.0 & \\
\hline \multirow[t]{2}{*}{7} & 141 & 0 & 2.0 & 3.0 & 0 \\
\hline & 141 & 2 & 3.0 & 3.5 & \\
\hline \multirow[t]{2}{*}{8} & 253 & 0 & 3.5 & 3.5 & 1 \\
\hline & 253 & 2 & 5.5 & 5.5 & \\
\hline \multirow[t]{2}{*}{9} & 353 & 0 & 2.0 & 1.0 & 0 \\
\hline & 353 & 2 & 2.5 & 1.5 & \\
\hline
\end{tabular}

${ }^{a}$ First two digits indicate tooth number according to the FDI World Dental Federation two-digit notation; third digit indicates site position: 1 - mesio-buccal, 3 - disto-buccal.

CAL: clinical attachment loss; PD: pocket depth.

generate libraries from amplified DNA. Normalized libraries were pooled and sequenced using the $2 \times 250$ Miseq v2 cartridge.

\section{Selection of genomes in databases}

Genomes of archaea and bacteria as well as their associated information were downloaded from the HOMD database server [26], the Pathosystems Resource Integration Center (PATRIC) ftp server [27,28], and the J. Craig Venter Institute [29]. A total of 524 genomes from 312 species of bacteria and two genomes from one archaea species were used in the analysis (Additional file 1: Table S7). Viral genomes were downloaded from NCBI [30].

\section{Short reads sequence alignment analysis}

Low-quality sequences were removed from the query files. Fast clipper and fastq quality filter from the Fastxtoolkit [31] were used to remove short sequences with quality score $>20$ in $>80 \%$ of the sequence. Cleaned files were then aligned against the bacterial/archaeal database using bowtie2. We generated a .gff file to map hits to different regions in the genomes of our database. Read 
counts from the SAM files were obtained using bedtools multicov from bedtools [32].

\section{Phylogenetic analysis of the metagenome and metatranscriptome}

Counts from the DNA and RNA libraries were used to determine the phylogenetic composition of the respective libraries. We created a .gff file containing information on whole genomes that was used to assign hits to genomes. Abundance estimation at the species level was performed applying the Genome Abundance Similarity Correction (GASiC) proposed by Lindner and Renard to estimate true genome abundances via read alignment by considering reference genome similarities in a nonnegative LASSO approach [33]. Estimated counts were normalized by frequency and $\log 2$ transformed before final analysis. To identify significant differences between communities under the different conditions studied we performed linear discriminant analysis (LDA) effect size (LEfSe) as proposed by Segata et al. [34], with default settings.

\section{Differential expression analysis}

For assessing differential expression in genes within a specific species we normalized the transcript counts by the relative frequency of the species in the metagenome database. In the case of Gene Ontology (GO) term analysis, we did not normalize by relative abundance since we were treating the whole community as a single organism. To identify differentially expressed genes from the RNA libraries, we applied non-parametric tests to the normalized counts using NOISeqBIO function of the $\mathrm{R}$ package NOISeq default conditions $(\mathrm{k}=0.5, \mathrm{lc}=1$, replicates $=$ 'biological' and rpkm normalization (rpkm option) using the threshold value for significance suggested by the authors of $q=0.95$, which for the function NOISeqBio is equivalent to an FDR cutoff of 0.05 [35,36].

\section{GO enrichment analysis}

To evaluate functional activities differentially represented in health or disease, we mapped the differentially expressed genes to known biological ontologies based on the GO project [37]. GO terms to which the different ORFs belong were obtained from the PATRIC database [27]. GO terms not present in the PATRIC database and whose annotation was obtained from the HOMD database or from the J. Craig Venter Institute were acquired using the program blast2GO under the default settings [38].

Enrichment analysis on these sets was performed using the $\mathrm{R}$ package GOseq, which accounts for biases due to over-detection of long and highly expressed transcripts [39]. Gene sets with $\leq 10$ genes were excluded from analysis. We used the REVIGO web page [40] to summarize and remove redundant GO terms from the results. Only GO terms with FDR $<0.05$ were used. REVIGO plots were obtained for biological processes categories.

\section{Quantification of putative virulence factors}

To identify putative virulence factors we used the Virulence Factors of Pathogenic Bacteria Database (VFDB) [41]. A similar approach, but with less stringent conditions, has been used by other authors to identify putative virulence factors in genomic islands [42]. The VFDB contains 1,205 virulence factors and 5,955 virulence factor related genes from 75 pathogenic bacterial genera [43]. We performed a BLAST similarity search of encoded proteins from the genomes in our database against the VFDB, with an e-value cutoff of 10 to 25 and identity $>99 \%$ to exclude distant homologs.

\section{Integration of metatranscriptomic results with clinical traits}

To integrate omics results with clinical parameters we used the $\mathrm{R}$ package mixOmics $[44,45]$. We calculated the sparse partial least square (sPLS) correlations between the clinical traits and species frequencies and profiles of gene expression in the progressing sites. Metatranscriptome hits were normalized by frequencies obtained in the metagenome before mixOmix analysis. For gene expression profiles, low count genes were filtered using the NOISeq function filtered.data with filter 1 and a minimum count per million of 30 [36]. Correlation Circle plots were obtained on the sPLS results to visualize associations between principal components species and gene expression profiles. Relevance networks showing correlations between genes and clinical traits were visualized in Cytoscape [46] with a threshold correlation of 0.95 .

\section{Results}

Phylogenetic differences between sites in metagenome and metatranscriptome composition

The comparison of phylogenetic assignments of the metagenome is presented in Figure 1. Two major observations can be derived from these results. First, changes in the metagenome of non-progressing sites were minor, only four species were significantly more abundant in the community at the endpoint of our study (Figure 1A). Second, differences in progressing sites were more significant (Figure 1B) and in those Streptococcus spp. dominated the community at baseline compared with the progressing community. What was more striking was the complete rearrangement at the metagenome level between the baselines of sites that did not progress versus sites that did progress (Figure 1C). Additionally, we compared the metagenome of baseline from progressing sites and non-progressing sites with samples from healthy sites of periodontally healthy individuals from a previous study [1]. The metagenome composition of 
A

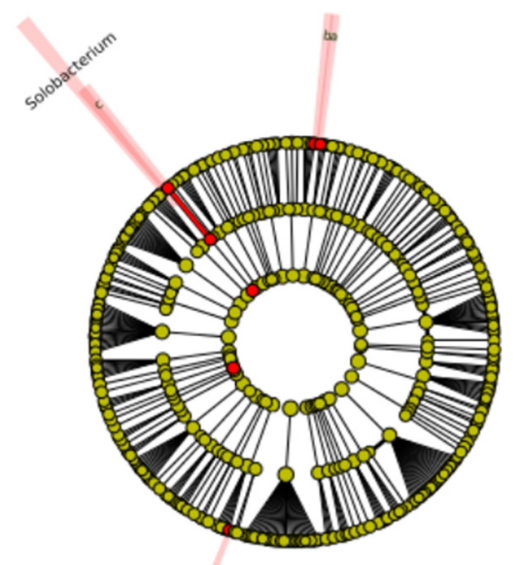

$\mathrm{B}$

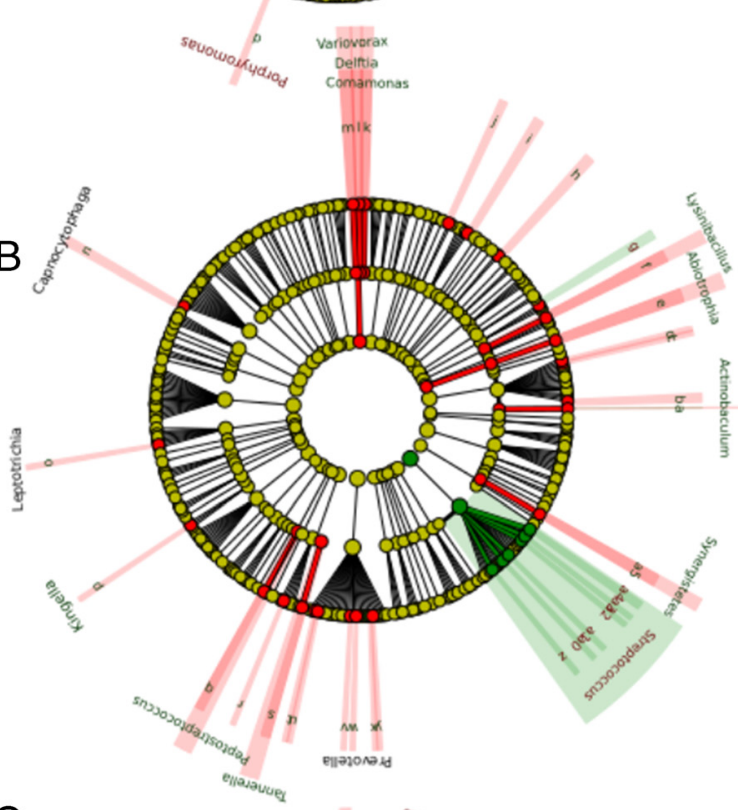

C

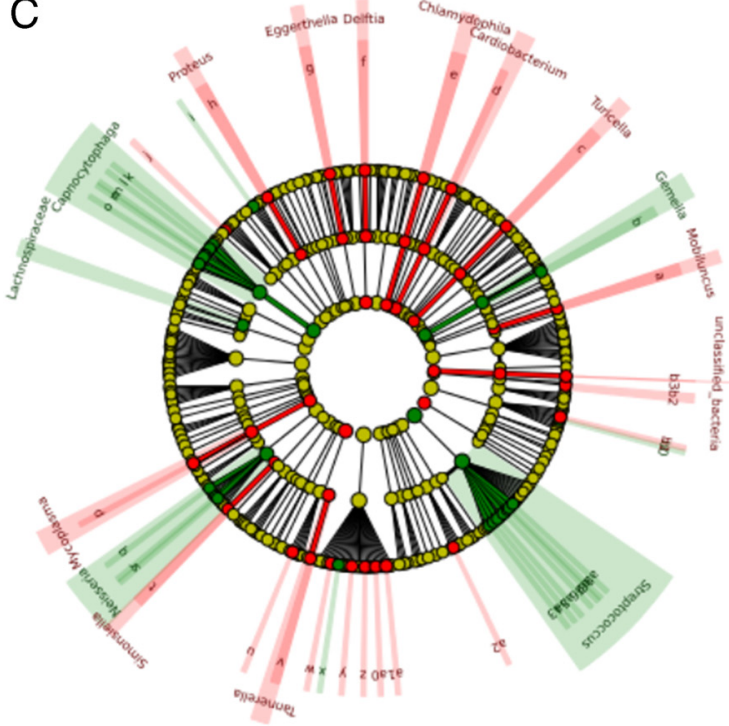

$\square$ Baseline

$\square$ Inactive sites

a: Parvimonas_sp_oral_taxon_393

b: Peptoniphilus_indolicus

c: Solobacterium_moorei

d: Porphyromonas_sp_oral_taxon_279 a: Mobiluncus mulieris c: Turicella_otitidis d: Cardiobacterium_valvarum e: Chlamydophila_pneumonia 1: Delfia_acidovorans 9: Eggerthella_lenta h: Proteus_mirabilis j: Eubacterium_yuri

p: Mycoplasma hominis t: Simonsiella muelleri u: Simonsiella_muelleri U. Uorphyromonas_catoniae v: Tannerella_sp_ora_taxon_BU063 w: Prevotella_histicola y: Prevotella_multisaccharivorax z: Prevotella_pallens a0: Prevotella_sp_oral taxon 302 a1: Prevotella_sp_oral taxon 473 a2: Treponema lecithinolyticum b1: Selenomonas_sp_oral taxon_. b2: Stenotrophomonas maltophilia b3: Candidate_division_SR1

\section{Baseline}

Active sites

$\square$ a: Actinobaculum_sp_oral_taxon_183 g: Gemella_sanguinis

2: Streptococcus_cristatus

$\square$ a: Streptococcus intermedius

a1: Streptococcus_oligofermentans

$\square$ a2: Streptococcus sanguinis

$\square$ a3: Streptococcus_sp_oral_taxon_56

a4: Streptococcus_vestibularis

Baseline active sites

Baseline inactive sites

Figure 1 (See legend on next page.) 
(See figure on previous page.)

Figure 1 Statistical differences in metagenome composition. Metagenome hit counts were first normalized using GASIC. Normalized counts were then analyzed using LEfSe with default parameters, to identify significant differences at species level between the microbial communities compared. (A) Comparison baseline samples from active sites vs. progressing samples from active sites (that is, samples collect at the visit when an increase in CAL $\geq 2 \mathrm{~mm}$ was detected). (B) Comparison baseline samples from stable sites vs. follow-up samples from stable sites (that is, collected 2 months after baseline). (C) Comparison baseline samples from active sites vs. baseline samples from stable sites.

both baseline communities is altered when compared to healthy communities (Additional file 2: Figure S1). Streptococcus spp. were more abundant in health than in the non-progressing baseline, while known periodontal pathogens such as $T$. denticola and $T$. forsythia were more abundant in the baseline samples (Additional file 2: Figure S1A). However, Streptococcus spp. were more abundant in the baseline from progressing sites than in healthy samples (Additional file 2: Figure S1B).

We then looked at the fraction of the active community under the conditions studied. To perform these analyses we first normalized the metatranscriptome results by the relative abundance of the different species to obtain differences in expression due to real changes in levels of gene expression and not to increases in numbers of certain members of the community. Species frequencies were estimated using GASIC [33]. Figure 2 shows the results of these analyses. As in the case of the metagenome, only a few species were significantly more active in the non-progressing sites at the end of the study (Figure 2A). When we compared progressing sites to their baseline, and baselines from progressing and non-progressing sites, the differences were larger (Figure 2B and C). Streptococcus spp. dominated the activity of the community at baseline of progressing sites (Figure 2B). Moreover, a member of the red complex, $P$. gingivalis, several members of the orange complex including $P$. intermedia and E. nodatum, and the putative periodontopathogen Filifactor alocis were more active at the baseline of active sites than the baseline of non-progressing sites (Figure 2C).

As with the metagenome, we also compared the results of activity at baseline and time of progression with the activity of the community in healthy samples. When comparing baseline of non-progressing sites to healthy sites, the first obvious results was that a larger fraction of the community is more active in the baseline samples than in health, even though they were clinically similar (Additional file 2: Figure S2). The same could be said for the baseline samples of sites that progressed; a large fraction of the community was significantly more active than the subgingival communities in health (Additional file 2: Figure S3).

Community-wide changes in patterns of gene expression in non-progressing and progressing sites during periodontitis progression

We first characterized differences in gene expression between baseline and progression in sites that showed disease activity during monitoring. We analyzed the global behavior of the community by identifying enrichment of $\mathrm{GO}$ terms. These results represent global changes in the community and could be due to overexpression of certain genes as well as increase in members of the community whose contribution to the observed activities is now higher due to their larger number. Potassium and amino-acid transport, peptidoglycan catabolism, isoprenoid biosynthesis, polysaccharide biosynthesis, and protein kinase $\mathrm{C}$-activating $\mathrm{G}$-protein coupled receptor signaling pathway were over-represented activities at baseline when compared to the end point of progression (Figure 3A). Over-represented activities at sampling time in progressing sites are shown in Figure 3B. We observed an over-representation of pathogenesis associated GO terms as well as activities related to response to oxidative stress.

We normalized the hit counts against species frequencies estimated using GASIC [33]. These normalized counts represented actual over-expression at the species level and not just increase in the total number of individuals in the community. Interestingly, the list of differentially expressed (DE) genes before and after normalization was very similar (Additional file 2: Figure S4). A total of 132,251 of the DE genes were identical in both, normalized and no-normalized gene sets; 412 DE genes were only identified exclusively in the normalized list and only 6,126 in the no-normalized set of $\mathrm{DE}$ genes. These results suggest that most of the differences observed were due to changes in gene expression at the species level rather than changes in the numbers of the members of the community.

When we compared the expression profiles of baseline and follow-up samples from non-progressing sites (that is, sites that did not change based on CALs) we did not identify any gene as differentially expressed, indicating that clinically stable sites did not have significant changes in gene expression during the period we studied those sites.

Changes in gene expression profiles in major periodontal pathogens members of the red complex ( $P$. gingivalis, $T$. denticola, and $T$. forsythia) during periodontal disease progression showed that upregulated genes belonged to GO terms associated with transport (iron, cation, lactate, citrate, sodium, and phosphate), proteolysis, protein kinase $\mathrm{C}$-activating G-protein coupled receptor signaling pathway and response to antibiotic (Additional file 2: Figure S5A), while downregulated genes belonged to GO terms associated with cobalamin (vitamin B12) biosynthesis (Additional 


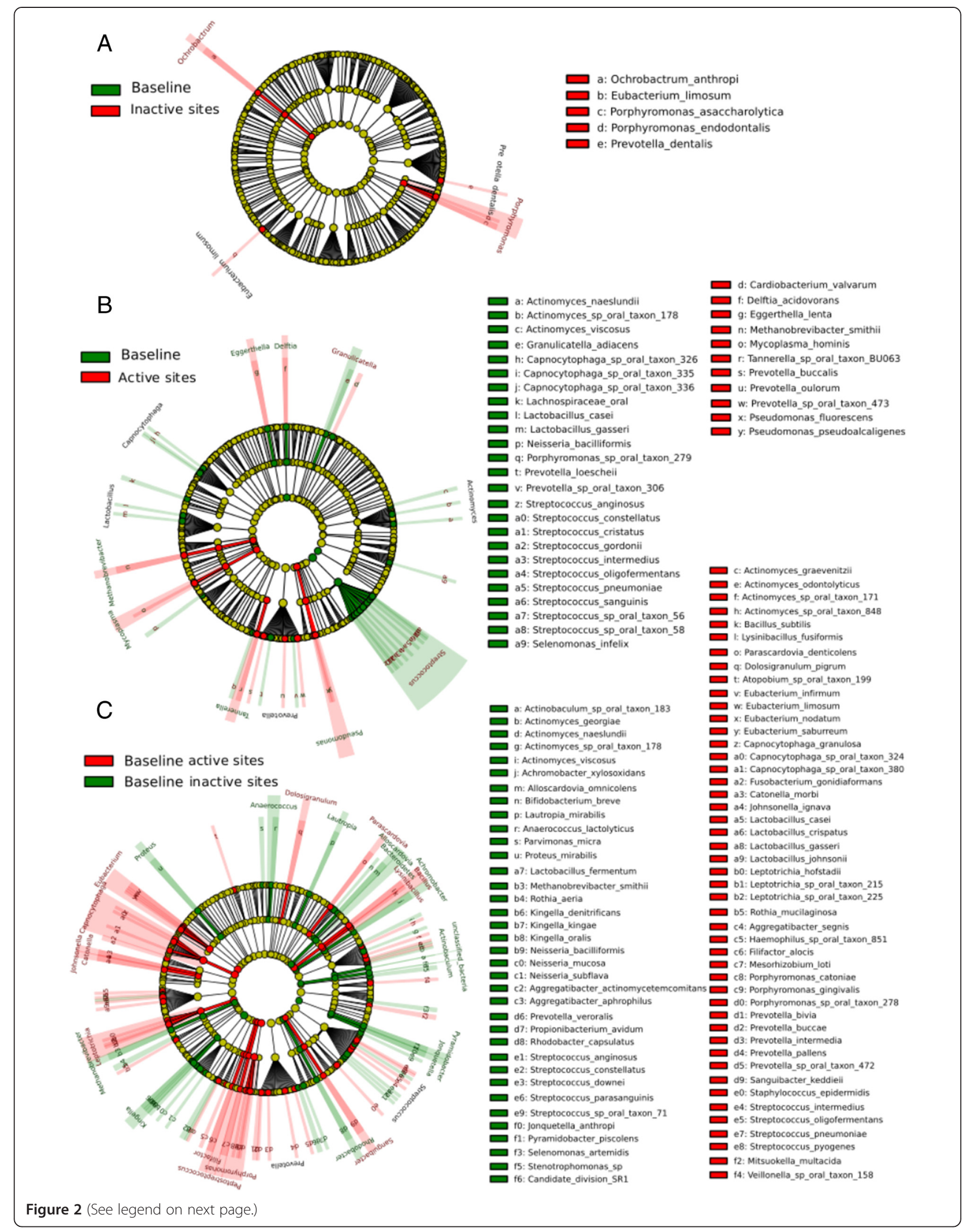


(See figure on previous page.)

Figure 2 Statistical differences in metatranscriptome normalized composition. Metatranscriptome hits were first normalized by the relative frequency of species obtained in the metagenomic analysis using GASIC. Normalized counts were then analyzed using LEfSe with default parameters to identify significant differences in activity at the species level. (A) Comparison baseline samples from active sites vs. progressing samples from active sites (that is, samples collect at the visit when an increase in CAL $\geq 2 \mathrm{~mm}$ was detected). (B) Comparison baseline samples from stable sites vs. follow-up samples from stable sites (that is, collected 2 months after baseline). (C) Comparison baseline samples from active sites vs. baseline samples from stable sites.

file 2: Figure S5B). Individually we observed that Treponema denticola upregulated genes related to flagella biosynthesis (flaA, fla G, fliQ, and fliW), oligopeptide $\mathrm{ABC}$ transporters, and a large number of hypothetical proteins (Additional file 3: Table S1). Tannerella forsythia and Porphyromonas gingivalis both upregulated different TonBdependent receptors, genes involved in iron transport (ferric uptake siderophores and ferrous iron transport protein B), a large number of peptidases and proteases including $\mathrm{ClpB}$, genes associated with aerotolerance (Bacteroides aerotolerance operon batA-E and moxR-like ATPase of the aerotolerance operon), and CRISPR- associated genes (csp, csm, and cas genes) (Additional file 3: Table S1).

Finally, $P$. gingivalis specifically upregulated genes related to biotin synthesis (biotin synthase, bioC, and
bioG), capsular polysaccharide biosynthesis proteins and a large number of proteins of conjugative transposons (tra $\mathrm{A}, \operatorname{tra} \mathrm{B}, \operatorname{tra\mathrm {E}}, \operatorname{tr} a \mathrm{~F}, \operatorname{tra} \mathrm{G}, \operatorname{tra} \mathrm{I}, \operatorname{tra} \mathrm{J}, \operatorname{tra} \mathrm{K}, \operatorname{tr} a \mathrm{~L}, \operatorname{tra\mathrm {M}}$, tra $\mathrm{N}$, traO, trap, and traQ) and transposases (ISPg2, ISPg3, ISPg4, ISPg5, and ISPg6). T. forsythia specifically upregulated transposases (IS116, IS110, IS902, and IS4 families) and large numbers of different homologs of SusC and SusD family proteins, involved in polysaccharide binding. Regarding downregulated proteins of the red complex most of them were hypothetical in all three of its members (Additional file 4: Table S2).

Profiles of expression of the members of the orange complex were very similar to the ones from the red complex. They upregulated different TonB-dependent receptors, a large number of peptidases and proteases

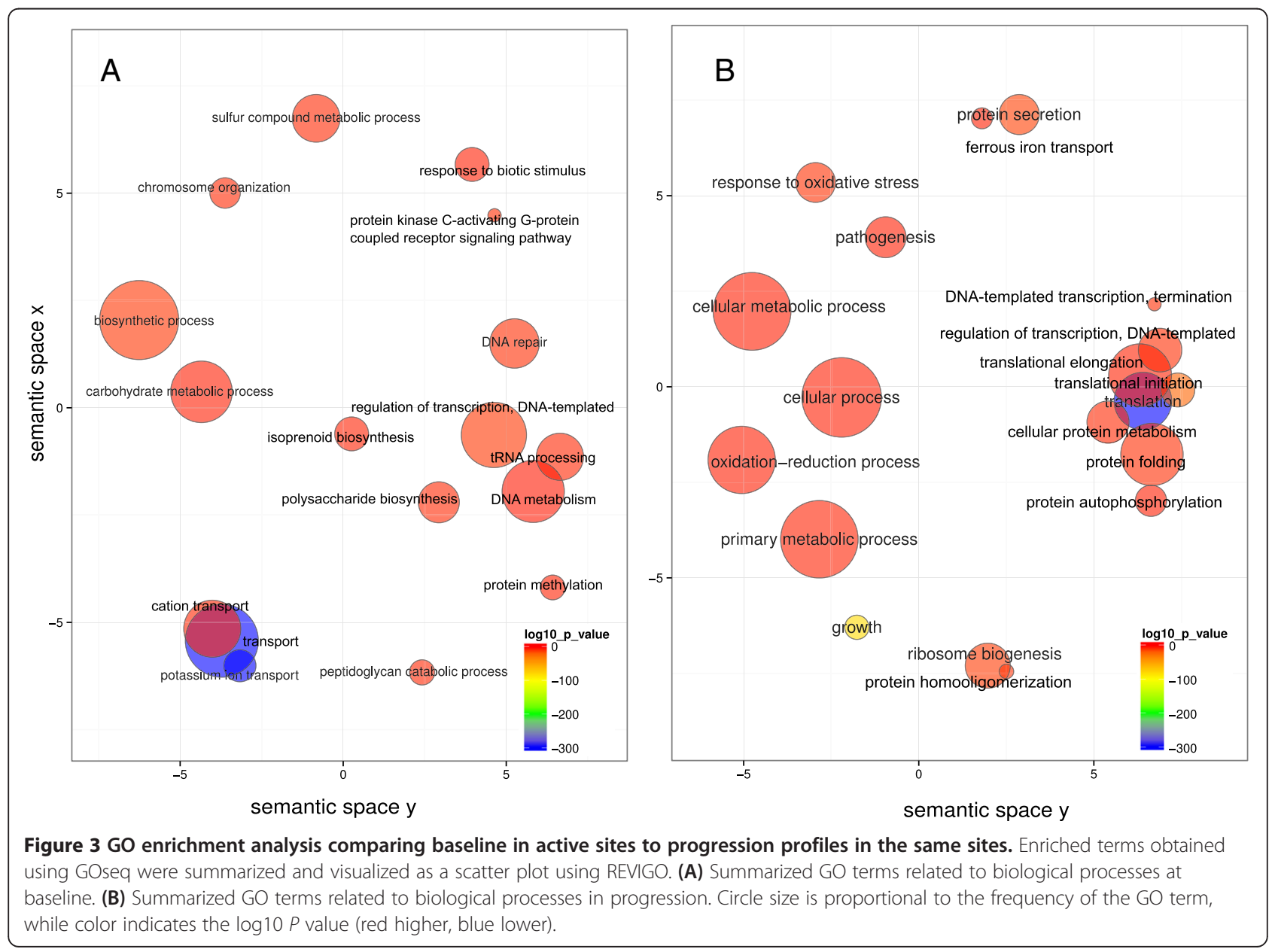


including $\mathrm{ClpB}$, genes associated with aerotolerance (Bacteroides aerotolerance operon batA-E $\mathrm{i}$ and mox Rlike ATPase of the aerotolerance operon in P. intermedia and $P$. nigrescens), genes involved in iron transport (ferric uptake siderophores and ferrous iron transport protein B), hemolysins, CRISPR- associated genes (in $C$. gracilis, C. rectus, C. showae, $P$. nigrescens, and $S$. constellatus) and chaperones GroEL, GroES, and GrpE (Additional file 5: Table S3). As in the case of $P$. gingivalis, both $P$. intermedia and $P$. nigrescens upregulated a large number of mobilization genes from conjugative transposons (tra $\mathrm{A}, \operatorname{tra} \mathrm{B}, \operatorname{tra} \mathrm{D}, \operatorname{tra} \mathrm{E}, \operatorname{tra} \mathrm{F}, \operatorname{tra} \mathrm{G}, \operatorname{tra} \mathrm{I}, \operatorname{tra} \mathrm{J}$, $\operatorname{tra\mathrm {K}}, \operatorname{tra} \mathrm{L} \operatorname{tra\mathrm {M}}, \operatorname{tra\mathrm {N}}, \operatorname{tra\mathrm {O}}$, and $\operatorname{tra} \mathrm{Q}$ ) (Additional file 5: Table S3).

\section{Comparison of metatranscriptomic profiles from progressing vs. non-progressing sites at baseline}

In order to identify activities that could be related to the initial steps of disease progression we compared community-wide expression profiles of samples at baseline from sites that did not progress vs. sites that did. The analysis of GO enrichment terms showed an over-representation in the baseline of progressing sites of terms related to cell motility, transport (iron, potassium, chloride, citrate, and amino acids transport), lipid A and peptidoglycan biosynthesis, protein kinase $\mathrm{C}$-activating G-protein coupled receptor signaling pathway, as well as synthesis of aromatic compounds (Figure 4A). On the other hand, in the baseline samples from non-progressing sites there was an overrepresentation of $\mathrm{GO}$ terms related to tricarboxylic acid cycle, metal ion transport, phosphoenolpyruvatedependent sugar phosphotransferase system and protein secretion (Figure 4B).

When compared baselines with healthy sites we found that the clinically healthy sites at baseline from diseased individuals were already impacted by disease. Both baselines from progressing and non-progressing sites had an over-representation of GO terms associated with citrate, organic ion, and lactate transport, as well as sulfur compound metabolic processes and peptidoglycan catabolism (Additional file 2: Figure S6).

Differences in gene expression of the red complex between baseline samples from progressing vs. non-
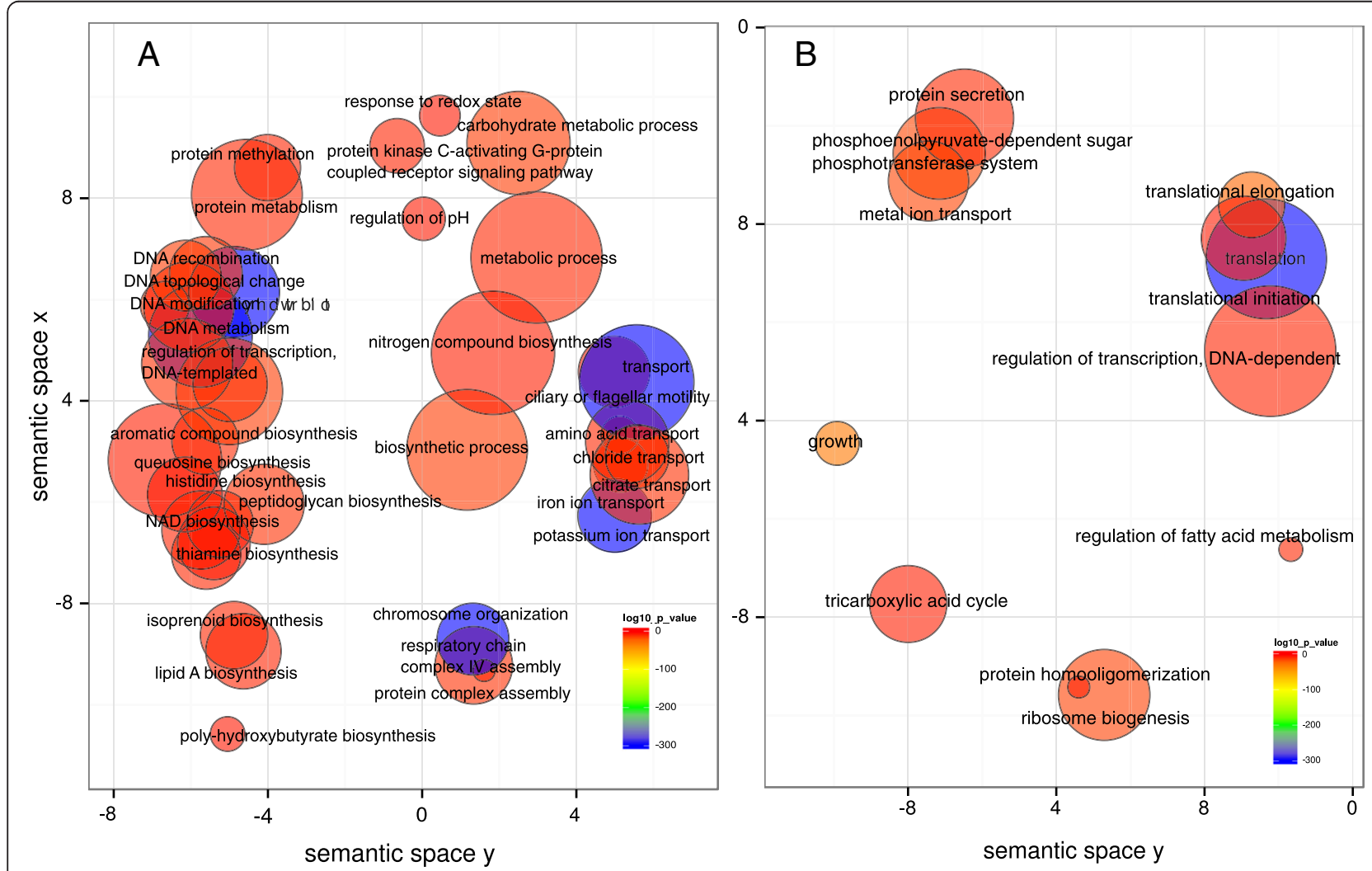

Figure $4 \mathrm{GO}$ enrichment analysis comparison of baselines from progressing and non-progressing sites. Enriched terms obtained using goseq were summarized and visualized as a scatter plot using REVIGO. (A) Summarized GO terms related to biological processes in baselines of progressing sites. (B) Summarized GO terms related to biological processes in baselines of non-progressing sites. Circle size is proportional to the frequency of the GO term, while color indicates the $\log 10 P$ value (red higher, blue lower). 
progressing showed similar enrichment of activities than when studying progressing sites. GO assignment of the differentially expressed genes showed association with proteolysis, sulfur compound metabolism and response to antibiotic in upregulated genes and translation in downregulated genes (Additional file 2: Figure S7). $P$. gingivalis actively upregulated putative virulence factors (34 in total) while $T$. denticola, with three upregulated putative virulence factors, and $T$. forsythia, with one, seem not especially active at this stage (Additional file 6: Table S4).

A more complex picture emerged when we analyzed the behavior of the orange complex. As a whole the members of the orange complex (P. intermedia, $P$. nigrescens, $P$. micra, F. nucleatum, $F$. periodonticum, $C$. gracilis, $C$. rectus, $S$. constellatus, $E$. nodatum, and $C$. showae) showed upregulation of genes associated with proteolysis, sodium ion transport, cellular response to phosphate starvation, and regulation of $\mathrm{pH}$ (Additional file 2: Figure S8A).
Expression of putative virulence factors in the oral community during periodontitis progression and at baseline of progressing vs. baseline of non-progressing sites

In the case of progressing sites, when comparing baseline vs. break down visit, a total of 9,147 hits of putative virulence factors from 207 species were identified in the genes over-represented in progressing samples. Nonetheless, not all of them expressed a large number of them, only 47 showed upregulation of 50 or more putative virulence factors under these conditions. Two members of the red complex, $P$. gingivalis and $T$. forsythia, expressed a large number of the putative virulence factors in the progressing samples (Figure 5A). More active in these samples were members of the orange complex: C. gracilis, F. nucleatum, P. intermedia, S. constellatus, $P$. nigrescens, and P. micra (Figure 5A). Three members of this complex were especially active in the upregulation of putative virulence factors: C. gracilis, F. nucleatum, and $P$. intermedia upregulated 114, 90, and 82 genes, respectively, that have homology with virulence

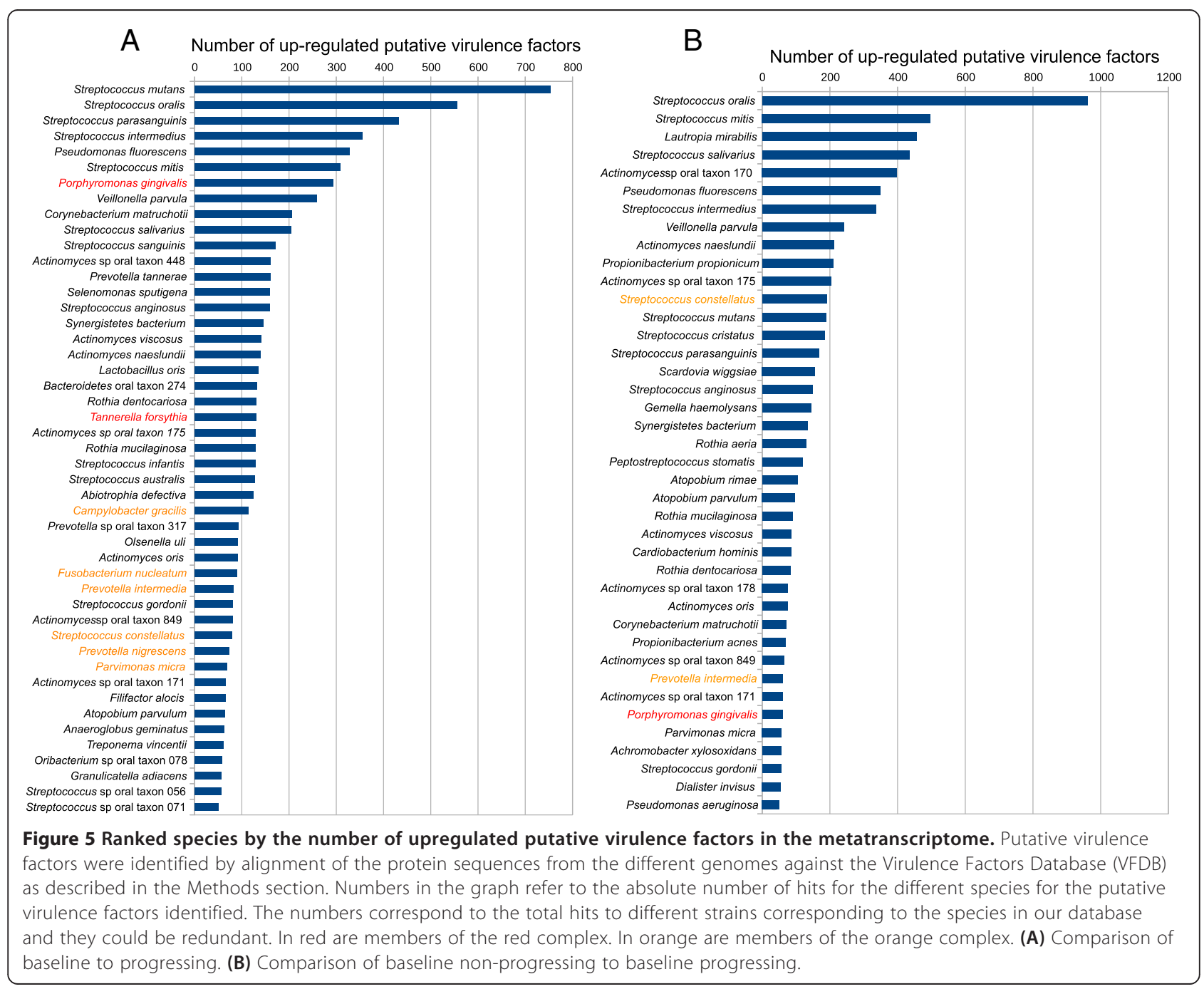


factors in our database (Additional file 7: Table S5). $P$. micra upregulated 69 putative virulence factors, $P$. nigrescens $73, S$. constellatus 79, C. rectus 13 , and C. showae 11 . We did not identify any putative virulence factor upregulated from $E$. nodatum or from $F$. periodonticum. When we looked at the global activities associated with the expression of these virulence factors we obviously observed an over-representation of GO terms related to pathogenesis as well as iron transport and lipid A biosynthesis (Additional file 2: Figure S9A). Focusing on the members of the red complex we could also see as common themes an up-regulation of genes associated with iron transport and lipid A biosynthesis (Additional file 2: Figure S9B). More interestingly were the results associated with the orange complex. In addition to the same activities mentioned for the red complex, members of the orange complex upregulated genes involved in cell adhesion, proteolysis, and pilus assembly during progression (Additional file 2: Figure S9C).

We then looked at the global activities associated with the expression of virulence factors comparing baselines of non-progressing vs. progressing sites and found that in the baseline of progressing sites the major activities corresponded to pathogenesis and ferrous ion transport while in non-progressing baselines there was an overrepresentation of GO terms related to cobalamin biosynthesis and sodium ion transport (Additional file 2: Figure S10). Comparing the expression of virulence factors at baseline gave a better understanding of the role of two complexes (red and orange) in the first stages of disease. We did not observe a significant over-representation of any GO terms in any of the members of the red complex. Nonetheless, members of the orange complex were indeed upregulating genes involved in proteolysis and iron homeostasis (Additional file 2: Figure S11). Surprisingly enough, we identified a set of organisms that were highly active, transcribing genes of putative virulence factors that have not been usually associated with periodontal disease. S. oralis, S. mutans, S. intermedius, S. mitis, $V$. parvula, and $P$. fluorenscens were upregulating a large number of putative virulence factors in both analysis (baseline vs. progression, comparing baseline in progression with baseline in non-progressing sites) (Figure 5).

All of the above cited organisms upregulated different oligopeptide transport systems (oppA, oppD, oppF, and oppB). S. oralis, S. mutans, S. intermedius, S. mitis, P. fluorenscens, and $V$. parvula upregulated several hemolysins, manganese $\mathrm{ABC}$ transporters, manganese superoxide dismutase, and a protein serine threonine phosphatase (PrpC) involved in regulation of stationary phase (Additional file 8: Table S6). S. oralis, S. mitis, and V. parvula upregulated vitamin B12 ABC transporters (Additional file 8: Table S6). S. oralis, S. mutans, S. intermedius, and S. mitis all upregulated Clp protease and LytR trancriptional attenuator. $P$. fluorescence upregulated all genes associated with flagellar synthesis $(f l a \mathrm{~A}, f l a \mathrm{G}, f l e \mathrm{~N}, f l g \mathrm{~A}, f l g \mathrm{C}, f l g \mathrm{~F}, f l g \mathrm{I}, f l g), f l g \mathrm{~K}, f l g \mathrm{~N}$, $f l h \mathrm{~A}, f l h \mathrm{~B}, f l h \mathrm{~F}, f l i \mathrm{D}, f l i \mathrm{~F}, f l i \mathrm{G}, f l i \mathrm{I}, f l i \mathrm{~K}, f l i \mathrm{~N}, f l i \mathrm{P}, f l i \mathrm{Q}, f l i \mathrm{R}$, $f l i \mathrm{~S}$, and $m o t \mathrm{~B}$ ) and genes related to chemotaxis (Additional file 8: Table S6).

In spite of the commonalities in upregulated genes when comparing baselines of progressing to nonprogressing sites and progression, there were also specific signatures of the two comparisons. For instance, $S$. oralis and $S$. mitis upregulated collagen adhesion proteins and $V$. parvula TonB-dependent receptors when comparing baselines but not during progression.

\section{Integrating expression profiles and clinical traits during periodontitis progression}

Integrating microbiological functions with clinical parameters is still one of the challenges in omics analysis. We used multivariate statistical analysis and visualization tools implemented in the $\mathrm{R}$ package mixOmics [44] to identify relevant associations between gene expression and certain clinical traits: bleeding on probing (BOP), increase in pocket depth $(\triangle \mathrm{PD})$ and increase in clinical attachment level $(\triangle \mathrm{CAL})$. We calculated the sparse partial least square (sPLS) correlations between the evolution of clinical traits and profiles of gene expression in the progressing sites. Additional file 2: Figure S12 visualizes of the relationships using correlation circle plots. Not surprisingly, $\triangle \mathrm{PD}$ and $\triangle \mathrm{CAL}$ were highly correlated between them (Additional file 2: Figure S12A). There was a set of genes whose expression profiles were highly correlated with $\triangle \mathrm{PD}$ and $\triangle \mathrm{CAL}$ evolution (Additional file 2: Figure S12A). There were no genes whose profiles correlated with BOP (Additional file 2: Figure S12). Interestingly, two large set of genes correlated with other components (Additional file 2: Figure S12B), but which possibly corresponded to another clinical trait not analyzed in our study.

We also analyzed correlation structures between clinical traits and gene expression using relevance networks [44]. This method generates a graph where nodes represent variables and the edges represent the correlations. The correlation of gene expression profiles gave a large number of genes that correlated with the clinical parameter profiles. Not surprisingly, BOP, which is a dichotomous variable with only two states (yes or no), did not correlate with any gene profiles. $\triangle \mathrm{PD}$ and $\triangle \mathrm{CAL}$ correlated with a large number of gene profiles, even with an $\mathrm{r}=0.95$. We then assigned GO terms to the correlated genes and summarized these results using REVIGO. We detected specific patterns of activities associated with increases in PD and CAL (Figure 6A and B). Among those patterns we found that the profiles of expression of phosphoenolpyruvate-dependent sugar phosphotransferase system, proteolysis and potassium transport were 


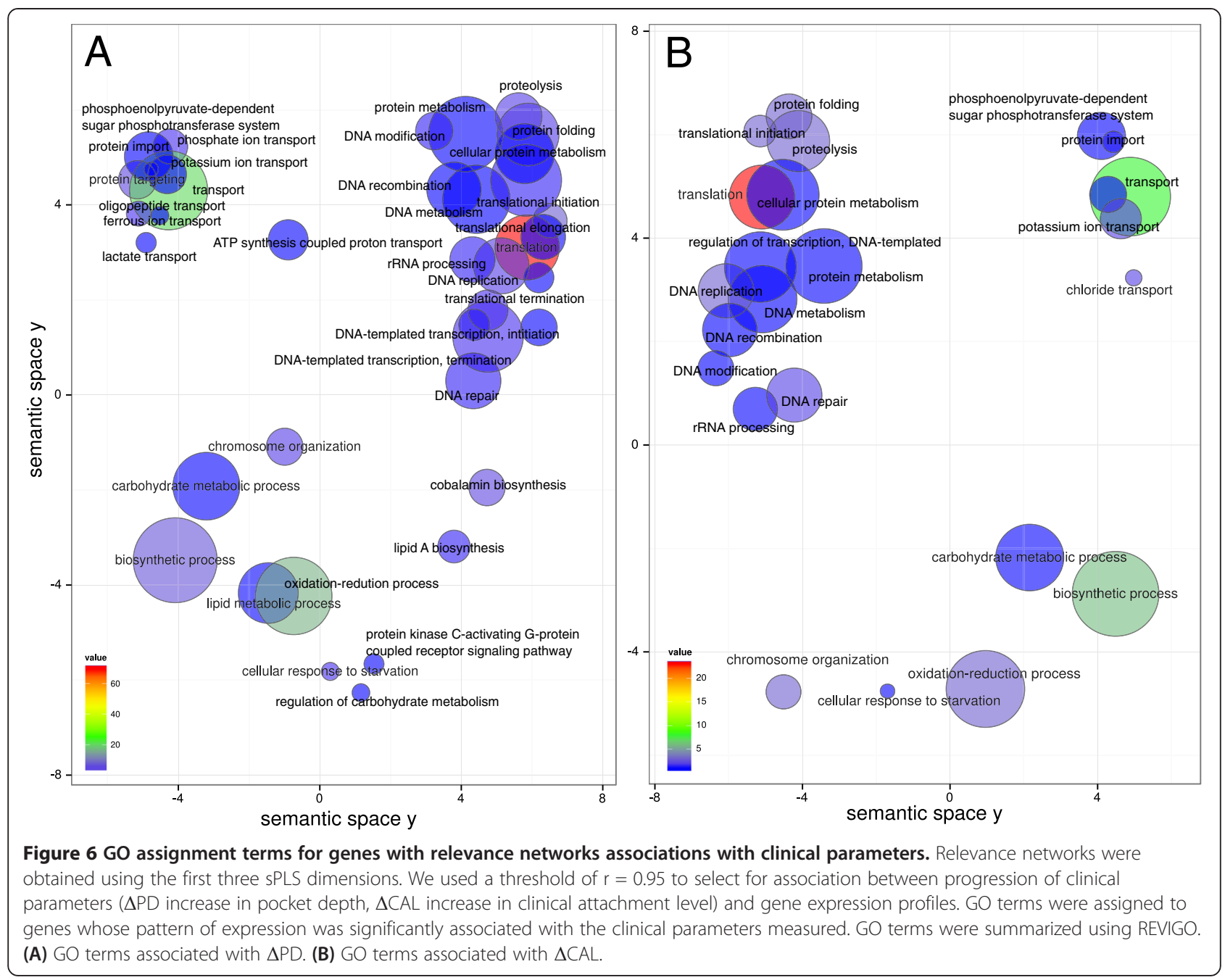

associated with the worsening of those two clinical parameters.

\section{Viral activity in the oral cavity during progression}

We also looked at the presence of eukaryotic viruses and bacteriophages, which have been previously associated with disease [47] and may play a role in shaping the bacterial community [48]. We identified viral activities in all samples although the number of transcripts represented a small fraction of all hits identified, between $0.04 \%$ and $0.7 \%$ of all hits were of viral origin (Additional file 2: Figure S13). To confirm that those hits really belong to viral sequences we obtained consensus sequences from the .bam files of our alignments and BLASTed them against the nr database at NCBI. Consensus sequence from 14 out of the 16 samples analyzed had sequences with significant matches to viral sequences. When we compared the relative activities of viruses at baseline and progression, we observed high activity of phages and herpesvirus in the progressing sites in relation with the baseline samples (Additional file 2: Figure S14).

\section{Discussion}

In a recent report we used a combined metagenomic/ metatranscriptomic approach to characterize the functional dysbiotic phenotype of the oral microbiome during severe chronic periodontitis. We took advantage of next generation sequencing (NGS) techniques and the fact that a large number of genomes from oral isolates have been sequenced [49] to infer functional differences between the subgingival microbiota of periodontal health and chronic severe periodontitis [1]. To date, only a few studies have been used to study the transcriptome of the community-wide oral microbiome, either to characterize microbial expression in caries [50,51] or periodontitis [52] but none of them addressed the question of why some dental site suffer peridontitis progression while other remain stable. 
Recent studies on community composition that used NGS analysis compared samples from periodontally healthy subjects with chronic periodontitis samples reached similar conclusions and suggested that members of the genera Prevotella, Fusobacterium, Treponema, Sinergistetes, Filifactor, and Porphyromonas and candidate division TM7 were more abundant in periodontitis whereas Actinomyces and Streptococcus were less frequent in samples from periodontitis compared to periodontally healthy subjects $[10,11]$. These results agreed with the associations of red and orange complexes with periodontal disease previously postulated using other detection methods $[9,53]$. We observed similar results in our previous study when comparing health and severe chronic periodontitis [1]. Although most analyses were performed using 16S rDNA sequencing and our current study used metagenomic analysis (shot gun sequencing using NGS), we also found that in the progressing sites Prevotella and Sinergistetes were more abundant when the teeth broke down than at baseline while the opposite was true for the genus Streptococcus. Non-progressing sites had almost the same metagenomic composition at baseline and at the 2-month visit, when another sample was taken.

Examining baseline samples might give us insights into the changes in microbial composition and activities that define the initial stages of progression. Large differences were observed when comparing baseline samples of progressing with non-progressing sites. Members of the genera Porphyromonas, Treponema, Tannerella, and Prevotella, among others, were more abundant at baseline on sites that progressed. Members of the genus Streptococcus were more abundant at baseline in nonprogressing sites.

We also looked at differences in the phylogenetic assignment of active members of the microbial community. We found that, as in the metagenome, no major differences were observed in stable sites when comparing baseline and 2-month visit samples. However, the differences where profound when we compared progressing sites at baseline with the breakdown time point and even more profound when we compared the two baseline samples from progressing and non-progressing sites. During progression Streptococcus spp. and Actinomyces spp. were highly active at baseline. In contrast Mycoplasma spp. were more active in progression. Although little attention has been paid to Mycoplasma spp. as potentially important in oral diseases in most studies, it has been significantly associated with periodontitis $[10,11,54,55]$. Interestingly, Synergistites sp. and the archaea Methanobrevibacter sp. were more active during progression. These microorganisms have been previously associated with periodontal disease $[10,11,56]$.

When we looked at differences at baseline between active and non-progressing sites we found that the known periodontal pathogens $T$. denticola and T. forsythia were not significantly more active while $P$. gingivalis was. $P$. gingivalis has been proposed as a keystone-pathogen in periodontitis. The keystone-pathogen hypothesis states that specific low-abundance microbes can lead the process of periodontal inflammation and tissue destruction by transforming a normally healthy microbiome into a dysbiotic state $[57,58]$. The high activity of $P$. gingivalis at baseline of active sites will agree with this hypothesis and it would indicate that even at early stages $P$. gingivalis is starting to be involved in the dysbiotic process that leads to progression.

Additionally, F. alocis, E. nodatum, and several Prevotella spp. were more active in the sites that would progress than in the site that would remain non-progressing. Both $F$. alocis and $E$. nodatum, a member of the orange complex, have been previously associated with periodontal disease [55,59-61].

Testing for differential representation of GO terms gives an overall view of the metabolic activities of the whole community under different environmental conditions. Interestingly, when we compared the expression profiles of longitudinal samples from stable sites, we did not identify any gene as differentially expressed. This indicates that the community as a whole did not change its expression patterns in the two months between the first visit and the time when subsequent samples were taken.

When we looked at progression, the results pointed to several functional signatures characteristic of the active sites. At the breakdown point active sites were actively expressing genes associated with pathogenesis, response to oxidative stress and ferrous iron transport. Oxidative stress is one of the consequences of the host inflammatory response to the microbial challenge [62] and bacteria must act to defense themselves against this host defense mechanism, which is probably accentuated with the progression of disease. Iron is an essential enzymatic cofactor and we have already shown the in situ overexpression of genes related to its transport in the microbial community during severe chronic periodontitis [1]. At baseline, GO terms associated with isoprenoid and polysaccharide biosynthesis, sulfur compound metabolic processes, potassium ion transport and protein kinase C-activating G-protein coupled receptor signaling pathway were highly enriched. Lipopolysaccharide (LPS) is a key factor in the development of periodontitis $[63,64]$ and high levels of lipopolysaccharide (LPS) from P. gingivalis have been reported to delay neutrophil apoptosis and provide a mechanism to modulate the restoration and maintenance of inflammation in periodontal tissues [65-67]. Hydrogen sulfide production from aminoacids and peptides has been reported in periodontal bacteria and the different efficiency of use of these compounds could be important determinants of the periodontal 
microbial ecology $[68,69]$. More puzzling is the overrepresentation of GO terms related to potassium transport. Potassium transport systems have been associated with pathogenesis in other organisms such as Staphylococcus aureus [70] and Salmonella [71], but not in oral bacteria. Interestingly, significant higher levels of potassium have been reported in periodontitis in both gingival crevicular fluid (GCF) and saliva [72,73].

Comparing baseline metabolic activities of nonprogressing vs. progressing sites might give us a better understanding of the initial stages of disease and the role that the microbial community plays at this early stage of pathogenesis. Among those functional signatures we found: citrate transport, iron transport, potassium transport, amino-acid transport, isoprenoid biosynthesis, and ciliary and flagellar motility. Citrate transport has been linked to iron transport and virulence in other organisms, such as Shigella and Pseudomonas [74,75]. This is in accordance with our previous observations in severe chronic periodontitis sites [1]. As mentioned above, the efficiency in utilizing various amino acids and peptides is among the key determinants of the periodontal microbial ecology [68] and its uptake may give additional advantages to certain members of the microbial community. In the active sites, there seems to be a shift from amino-acid uptake to oligopeptide uptake throughout the breakdown process. Isoprenoid biosynthesis, probably involved in the synthesis of peptidoglycan, was also over-represented in active sites. Isoprenoids are a large, diverse class of naturally occurring organic chemicals which are essential for cell survival. The $2 \mathrm{C}$ methyl-D-erythritol 4-phosphate (MEP) pathway has been implicated in the virulence of Listeria monocytogenes, Mycobacterium tuberculosis, and Brucella abortus [76], and evidence suggests that the MEP pathway may be involved in intracellular survival by combating oxidative stress [77]. Moreover, a metagenomic analysis of the human distal gut microbiome revealed that MEP pathway genes are highly abundant in that community; perhaps reflecting the abundance of the MEP pathway in bacteria in general [78]. Finally, ciliary and flagellar motility as well as chemotaxis genes that could direct bacterial movement were all part of the signature activities at the initial stages of progression. Motile pathogenic members of the oral community, such as Treponema spp., possess the capacity for tissue invasion thanks to the synthesis of flagella $[79,80]$; our results suggest that this fraction of the community is already active at the initial stages of progression.

Historically, members of the red and orange complexes have been associated with chronic periodontitis $[7,9]$. Consistent with their postulated role in progressing sites, we observed a high level of expression of putative virulence factors by members of both complexes when breakdown was clinically detected. However, at the baseline of our studies it seems that the relative importance of these complexes in the active sites is reduced. Only $P$. gingivalis, S. constellatus, and P. intermedia were actively expressing putative virulence factors.

Interestingly, members of the red complex showed enrichment in response to antibiotics (beta-lactamase activity) during progression and even at baseline of progressing sites. We observed the same phenomenon at whole community-level in our previous study comparing healthy sites vs. chronic severe periodontitis [1]. Betalactamase activity has been observed in adult periodontitis at low-level enzymatic activity but with high prevalence [81] and seems to be a frequent phenomenon in samples from polymicrobial diseases [82]. We still do not know what role this enzymatic activity plays on the progression of the disease given that the patients of this study were not treated with antibiotics at the time of sampling.

CRISPR- associated genes in P. gingivalis, T. forsythia, C. gracilis, C. rectus, C. showae, $P$. nigrescens, and S. constellatus were highly upregulated during progression. We observed phage activity in all samples analyzed, which could explain the high level of production of CRISPR-associated proteins as a mechanism of defense against viral activity [83]. However, we cannot discard the possibility that CRISPR-associated proteins are playing a broader role in the virulence mechanisms of periodontitis. Thus, recently CRISPR-Cas systems have been linked to stress responses and virulence in bacteria [84] and to competitive interactions between members of the red complex [85].

$P$. gingivalis, $P$. nigrescens, and $P$. intermedia upregulated all the traA-Q and mob genes in their chromosomal conjugative transposons. These genes are required for formation of a conjugal pore and DNA mobilization $[86,87]$. The upregulation of these genes could indicate conjugative transposons mobilization in Porphyromonas and Prevotella which would agree with evidence of natural horizontal transfer of antibiotic resistance through conjugative transposon mobilization in those organisms [87-89]. However, the mobilization of those conjugative transposons was most likely driven by the presence of antibiotics. In the current study, subjects did not use systemic antibiotics during the monitoring period when samples were collected. Therefore, it is not clear what signal(s) triggered this mobilization of conjugative transposons. It is noteworthy that we did not observe this phenomenon in severe chronic periodontitis samples [1].

The idea that the whole community acts as a pathogen rather than only specific organisms has been gaining traction in recent years $[1,90,91]$. In agreement with this hypothesis we found that a group of organism not usually considered pathogens were upregulating a large 
number of putative virulence factors in active sites. Among these groups, we observed that some streptococci, including S. mitis and S. intermedius, were especially active. Although $S$. mitis and S. intermedius are usually associated with periodontal health, they have also been found to form part of the community in periodontitis $[10,11]$. We found $V$. parvula highly active in both progressing and baseline sites, which was surprising since $V$. parvula is almost always associated with a healthy community $[10,23]$. However, streptococci and $V$. parvula have been identified as part of a cluster associated with periodontitis in adolescents [92]. Another surprising finding was the identification of $P$. fluorescens as one of the top producers of virulence factors. This is not an organism usually associated with periodontitis, although another member of its genus, $P$. aeruginosa, has indeed been associated with other important pathologies such as cystic fibrosis $[93,94]$. In our previous study on chronic severe periodontitis, we also observed a similar behavior where the whole community, and not only known periodontal pathogens, expressed more putative virulence factors in diseased sites. Among the most active producers of putative virulence factors was Corynebacterium matruchotii [1], which has also been associated with periodontitis in microbiome studies. Interestingly, we also found this organism as highly active in progressing sites but not at the baseline, indicating a shift into a pathogenic microbial community.

We then established association between profiles of clinical parameters such as $\mathrm{BOP}, \triangle \mathrm{PD}$, and $\triangle \mathrm{CAL}$ with profiles gene expression. BOP showed no association with changes in gene expression profiles. This is not surprising since BOP is a discrete variable. Nonetheless, $\triangle \mathrm{PD}$ and $\triangle \mathrm{CAL}$ were highly associated with proteolytic activity and potassium ion transport. Additionally, $\triangle \mathrm{PD}$ was associated with cobalamin biosynthesis and ferrous and oligopeptide transport. Proteolysis has been recognized as an important virulence determinant in periodontitis progression $[95,96]$. In the case of vitamin B12 (cobalamin) synthesis, we observed an upregulation of the vitamin $\mathrm{B} 12 \mathrm{ABC}$ transporter btuFCD system in $P$. gingivalis and $T$. forsythia and some members of the orange complex. $P$. gingivalis harbors all the genes necessary to convert precorrin-2 into cobalamin, but it lacks the genes for the synthesis of precorrin-2 [97]. In our previous study on chronic severe periodontitis we also observed an up-regulation of btuFCD system in $P$. gingivalis and $T$. forsythia [1]. An increase in synthesis and release to the external medium by other organisms of cobalamin might give members of the red and orange complex an ecological advantage if they start scavenging it.

\section{Conclusions}

The initial causes for transition from a healthy microbial community to a dysbiotic one are still not well understood in great part due to the complexity of the oral community. Using a metagenomic/metatranscriptomic approach, and comparing baseline samples from the same individuals, we have begun the study of the physiological changes in the microbial community that are associated with the initial stages of dysbiosis. Here, we show that in periodontitis progression there are certain characteristic activities that are associated with the onset of breakdown in specific teeth. Among those we found that citrate transport, iron transport, potassium transport, amino-acid transport, isoprenoid biosynthesis, and ciliary and flagellar motility are signatures of the initiation of periodontitis progression.

The data presented here illustrate that regardless of the overall composition of the community, certain metabolic signatures are consistent with disease and progression. For instance, the community composition in the progressing active sites was relatively different from the Massachusetts in chronic severe periodontitis sites previously described [1]. Nonetheless, in both cases we observed that iron transport and protein secretion activities are highly associated with advance stages of disease. Moreover, as we have shown in our previous study on chronic severe periodontitis [1], our results show that the whole community, and not just a handful of oral pathogens, is responsible for an increase in virulence that could lead to progression. Finally, we found that certain ecological changes could explain the evolution of certain clinical parameters. As we discuss above, an increase in production of cobalamin could exacerbate the growth of periodontal pathogens that lack the capabilities to synthesis this compound and explain, at least in part, the association of these organisms with increase in disease severity.

\section{Availability of supporting data}

The datasets used in these analyses were deposited at the Human Oral Microbiome Database (HOMD) under the submission numbers 20130522 [98] and 20141024 [99].

\section{Additional files}

Additional file 1: Table S7. List of genomes used in the study. We generated a database of genomes that contains 524 genomes from 312 species of Bacteria and one species of Archaea and was used for alignment of the sequences in our results.

Additional file 2: Figure S1. Statistical differences in metagenome composition. Figure $\mathbf{S} 2$. Statistical differences in normalized metatranscriptome composition comparing non-progressing sites baselines to healthy sites of healthy patients. Figure S3. Statistical differences in normalized metatranscriptome composition comparing progressing sites baselines to healthy sites of healthy patients. Figure S4. Venn diagram showing overlapping differentially expressed genes with and without normalization. Figure S5. GO terms associated with changes in gene expression profiles in major periodontal pathogens members of the red complex during periodontal disease progression. Figure S6. GO enrichment analysis comparing healthy sites from healthy individuals and baselines in progressing sites and non-progressing sites. Figure S7. GO terms associated with changes in gene expression profiles in major 
periodontal pathogens members of the red complex when comparing baselines of progressing and non-progressing sites. Figure $\mathbf{S 8}$. GO terms associated with changes in gene expression profiles in members of the orange complex when comparing baselines of progressing and non-progressing sites. Figure S9. GO terms associated with changes in gene expression of putative virulence factors in the oral community during periodontitis progression. Figure $\mathbf{S 1 0}$. GO terms enrichment analysis of virulence factors comparing baselines. Figure S11. GO terms enrichment analysis of virulence factors in the orange complex comparing baselines. Figure S12. Correlation circle plots of SPLS analysis. Figure S13. Percentage of hits corresponding to viral sequences. Figure S14. Statistical differences in viral composition of transcript libraries.

Additional file 3: Table S1. Upregulated genes in members of the red complex during periodontal disease progression. After identifying upregulated genes of the whole community we selected those upregulated genes corresponding to the red complex (Porphyromonas gingivalis, Treponema denticola, and Tannerella forsythia), which are consider major periodontal pathogens.

Additional file 4: Table S2. Downregulated genes in members of the red complex during periodontal disease progression. After identifying downregulated genes of the whole community we selected those downregulated genes corresponding to the red complex (Porphyromonas gingivalis, Treponema denticola, and Tannerella forsythia), which are consider major periodontal pathogens.

Additional file 5: Table S3. Differentially expressed genes in members of the orange complex. Members of the orange complex (P. intermedia, P. nigrescens, P. micra, F. nucleatum, F. periodonticum, C. gracilis, C. rectus, S. constellatus, E. nodatum, and C. showae) have been also associated with chronic periodontitis. Here were showed DE genes of the members of this complex during periodontal disease progression.

Additional file 6: Table S4. Differentially expressed (DE) putative virulence factors of the red complex when comparing the baseline expression profiles of active and inactive sites. Based on the VFDB (see Methods) we compared expression of DE putative virulence factors at baseline and selected those corresponding to the red complex.

Additional file 7: Table S5. Differentially expressed (DE) putative virulence factors of the orange complex when comparing the baseline expression profiles of active and inactive sites. Based on the VFDB (see Methods) we compared expression of DE putative virulence factors at baseline and selected those corresponding to the orange complex.

Additional file 8: Table S6. Upregulated virulence factors in highly active members of the community in progressing sites. Other members of the community, usually not considered pathogens, showed a large number of putative virulence factors being upregulated during periodontitis progression and at baseline of progressing sites. Here we summarized the results showing the species whose upregulation of putative virulence factors was high, along with locus ID and annotation of the genes according to PATRIC (See Methods).

\section{Competing interests}

The authors declare that they have no competing interests.

\section{Authors' contributions}

SY and AD-P contributed equally to this paper. They performed the laboratory experiments prior to sequencing. RT was responsible for sample collection and clinical assessments. KK performed all sequencing and JF-L was responsible for designing the experiments, data analysis, and writing of the manuscript. All authors reviewed the manuscript prior to submission. All authors read and approved the final manuscript.

\section{Acknowledgments}

We are grateful to Moira Cahan for reviewing the manuscript and her useful comments. This research reported was supported by the National Institute of Dental and Craniofacial Research of the National Institutes of Health (NIDCR/NIH) under award numbers DE021553 and DE021127.

\section{Funding}

Research reported in this publication was supported by the National Institute of Dental and Craniofacial Research of the National Institutes of Health under award numbers DE021553 and DE021127.

\section{Author details}

${ }^{1}$ Forsyth Institute, 245 First Street, Cambridge, Massachusetts 02142, USA. ${ }^{2}$ Harvard School of Dental Medicine, 188 Longwood Ave, Boston, MA 02115, USA. ${ }^{3}$ University of North Carolina Chapel Hill, School of Dentistry, Chapel Hill, NC 27599-7450, USA.

Received: 5 December 2014 Accepted: 9 March 2015

Published online: 27 April 2015

\section{References}

1. Duran-Pinedo AE, Chen T, Teles R, Starr JR, Wang X, Krishnan K, et al. Community-wide transcriptome of the oral microbiome in subjects with and without periodontitis. ISME J. 2014;8:1659-72.

2. Hajishengallis $\mathrm{G}$. The inflammophilic character of the periodontitisassociated microbiota. Mol Oral Microbiol. 2014;29:248-57.

3. Kassebaum NJ, Bernabé E, Dahiya M, Bhandari B, Murray CJL, Marcenes W. Global burden of severe periodontitis in 1990-2010: a systematic review and meta-regression. J Dent Res. 2014;93:1045-53.

4. Brown LJ, Johns BA, Wall TP. The economics of periodontal diseases. Periodontol 2000. 2002;29:223-34.

5. Eke PI, Dye BA, Wei L, Thornton-Evans GO, Genco RJ. Prevalence of periodontitis in adults in the United States: 2009 and 2010. J Dent Res. 201291:914-20

6. Seymour GJ, Ford PJ, Cullinan MP, Leishman S, Yamazaki K. Relationship between periodontal infections and systemic disease. Clin Microbiol Infect. 2007;13:3-10.

7. Socransky SS, Haffajee AD, Cugini MA, Smith C, Kent RLJ. Microbial complexes in subgingival plaque. J Clin Periodontol. 1998;25:134-44.

8. Holt SC, Ebersole JL. Porphyromonas gingivalis, Treponema denticola, and Tannerella forsythia: the "red complex", a prototype polybacterial pathogenic consortium in periodontitis. Periodontol 2000. 2005;38:72-122.

9. Socransky SS, Haffajee AD. Periodontal microbial ecology. Periodontol 2000. 2005;38:135-87.

10. Abusleme L, Dupuy AK, Dutzan N, Silva N, Burleson JA, Strausbaugh LD, et al. The subgingival microbiome in health and periodontitis and its relationship with community biomass and inflammation. ISME J. 2013;7:1016-25.

11. Griffen AL, Beall CJ, Campbell JH, Firestone ND, Kumar PS, Yang ZK, et al. Distinct and complex bacterial profiles in human periodontitis and health revealed by 165 pyrosequencing. ISME J. 2012;6:1176-85.

12. Goodson JM, Tanner AC, Haffajee AD, Sornberger GC, Socransky SS. Patterns of progression and regression of advanced destructive periodontal disease. J Clin Periodontol. 1982;9:472-81.

13. Heitz-Mayfield LJA. Disease progression: identification of high-risk groups and individuals for periodontitis. J Clin Periodontol. 2005;32:196-209.

14. Ricci M, Garoia F, Tabarroni C, Marchisio O, Barone A, Genovesi A, et al. Association between genetic risk score and periodontitis onset and progression: a pilot study. Arch Oral Biol. 2011;56:1499-505.

15. Eley BM, Cox SW. A 2-year longitudinal study of elastase in human gingival crevicular fluid and periodontal attachment loss. J Clin Periodontol. 1996;23:681-92.

16. Eley BM, Cox SW. The relationship between gingival crevicular fluid cathepsin B activity and periodontal attachment loss in chronic periodontitis patients: a 2-year longitudinal study. J Periodontal Res. 1996;31:381-92.

17. Khalaf H, Lönn J, Bengtsson T. Cytokines and chemokines are differentially expressed in patients with periodontitis: possible role for TGF- $\beta 1$ as a marker for disease progression. Cytokine. 2014;67:29-35.

18. Persson GR, Matuliené G, Ramseier CA, Persson RE, Tonetti MS, Lang NP. Influence of interleukin-1 gene polymorphism on the outcome of supportive periodontal therapy explored by a multi-factorial periodontal risk assessment model (PRA). Oral Health Prev Dent. 2003;1:17-27.

19. Charalampakis $G$, Dahlén $G$, Carlén $A$, Leonhardt A. Bacterial markers vs. clinical markers to predict progression of chronic periodontitis: a 2-yr prospective observational study. Eur J Oral Sci. 2013;121:394-402. 
20. Byrne SJ, Dashper SG, Darby IB, Adams GG, Hoffmann B, Reynolds EC. Progression of chronic periodontitis can be predicted by the levels of Porphyromonas gingivalis and Treponema denticola in subgingival plaque. Oral Microbiol Immunol. 2009:24:469-77.

21. Haffajee AD, Socransky SS. Attachment level changes in destructive periodontal diseases. J Clin Periodontol. 1986;13:461-75.

22. Haffajee AD, Socransky SS, Smith C, Dibart S. Microbial risk indicators for periodontal attachment loss. J Periodontal Res. 1991;26:293-6.

23. Dzink JL, Socransky SS, Haffajee AD. The predominant cultivable microbiota of active and inactive lesions of destructive periodontal diseases. J Clin Periodontol. 1988;15:316-23.

24. Hart SN, Therneau TM, Zhang Y, Poland GA, Kocher J-P. Calculating sample size estimates for RNA sequencing data. J Comput Biol. 2013;20:970-8.

25. Li H, Handsaker B, Wysoker A, Fennell T, Ruan J, Homer N, et al. The sequence alignment/Map format and SAMtools. Bioinformatics. 2009;25:2078-9.

26. The Human Oral Microbiome Database. [http://www.homd.org/]

27. PATRIC. Pathosystems Resource Integration Center. [www.patricbrc.org/ portal/portal/patric/Home]

28. Wattam AR, Abraham D, Dalay O, Disz TL, Driscoll T, Gabbard JL, et al. PATRIC, the bacterial bioinformatics database and analysis resource. Nucleic Acids Res. 2014;42:D581-91.

29. J. Craig Venter Institute. [www.jcvi.org]

30. NCBI FTP site for Viral Genomes. [ftp://ftp.ncbi.nlm.nih.gov/genomes/ Viruses/]

31. FASTX-Toolkit. FASTQ/A short-reads pre-processing tools. [hannonlab.cshl. edu/fastx_toolkit/]

32. Quinlan AR, Hall IM. BEDTools: a flexible suite of utilities for comparing genomic features. Bioinformatics. 2010;26:841-2.

33. Lindner MS, Renard BY. Metagenomic abundance estimation and diagnostic testing on species level. Nucleic Acids Res. 2013;41:e10.

34. Segata N, Izard J, Waldron L, Gevers D, Miropolsky L, Garrett WS, et al. Metagenomic biomarker discovery and explanation. Genome Biol. 2011;12:R60

35. Soneson C, Delorenzi M. A comparison of methods for differential expression analysis of RNA-seq data. BMC Bioinformatics. 2013;14:91

36. Tarazona S, García-Alcalde F, Dopazo J, Ferrer A, Conesa A. Differential expression in RNA-seq: a matter of depth. Genome Res. 2011:21:2213-23.

37. The Gene Ontology Consortium. [http://www.geneontology.org/]

38. Götz S, García-Gómez JM, Terol J, Williams TD, Nagaraj SH, Nueda MJ, et al. High-throughput functional annotation and data mining with the Blast2GO suite. Nucleic Acids Res. 2008;36:3420-35.

39. Young MD, Wakefield MJ, Smyth GK, Oshlack A. Gene ontology analysis for RNA-seq: accounting for selection bias. Genome Biol. 2010;11:R14.

40. Supek F, Bošnjak M, Škunca N, Šmuc T. REVIGO summarizes and visualizes long lists of gene ontology terms. PLoS One. 2011;6:e21800

41. Virulence Factors of Pathogenic Bacteria Database. [http://www.mgc.ac.cn/FF/

42. Ho Sui SJ, Fedynak A, Hsiao WWL, Langille MGl, Brinkman FSL. The association of virulence factors with genomic islands. PLoS One. 2009:4:e8094.

43. Chen L, Xiong Z, Sun L, Yang J, Jin Q. VFDB 2012 update: toward the genetic diversity and molecular evolution of bacterial virulence factors. Nucleic Acids Res. 2012;40:D641-5.

44. González I, Cao K-AL, Davis MJ, Déjean S. Visualising associations between paired "omics" data sets. BioData Min. 2012;5:19.

45. Liquet B, Lê Cao K-A, Hocini H, Thiébaut R. A novel approach for biomarker selection and the integration of repeated measures experiments from two assays. BMC Bioinformatics. 2012;13:325.

46. Smoot ME, Ono K, Ruscheinski J, Wang P-L, Ideker T. Cytoscape 2.8: new features for data integration and network visualization. Bioinformatics. 2011:27:431-2.

47. Slots J. Human viruses in periodontitis. Periodontol 2000. 2010;53:89-110

48. Pride DT, Salzman J, Haynes M, Rohwer F, Davis-Long C, White RA, et al. Evidence of a robust resident bacteriophage population revealed through analysis of the human salivary virome. ISME J. 2012;6:915-26.

49. Chen T, Yu W-H, Izard J, Baranova OV, Lakshmanan A, Dewhirst FE. The Human Oral Microbiome Database: a web accessible resource for investigating oral microbe taxonomic and genomic information. Database J Biol Databases Curation. 2010;2010:baq013.

50. Peterson SN, Meissner T, Su Al, Snesrud E, Ong AC, Schork NJ, et al. Functional expression of dental plaque microbiota. Front Cell Infect Microbiol. 2014;4:108
51. Benítez-Páez A, Belda-Ferre P, Simón-Soro A, Mira A. Microbiota diversity and gene expression dynamics in human oral biofilms. BMC Genomics. 2014;15:311.

52. Jorth P, Turner KH, Gumus P, Nizam N, Buduneli N, Whiteley M. Metatranscriptomics of the human oral microbiome during health and disease. mBio. 2014;5:e01012-4

53. Haffajee AD, Socransky SS, Patel MR, Song X. Microbial complexes in supragingival plaque. Oral Microbiol Immunol. 2008;23:196-205.

54. Kumar PS, Mason MR, Brooker MR, O'Brien K. Pyrosequencing reveals unique microbial signatures associated with healthy and failing dental implants. J Clin Periodontol. 2012;39:425-33.

55. Colombo APV, Boches SK, Cotton SL, Goodson JM, Kent R, Haffajee AD, et al. Comparisons of subgingival microbial profiles of refractory periodontitis, severe periodontitis, and periodontal health using the human oral microbe identification microarray. J Periodontol. 2009;80:1421-32.

56. Lepp PW, Brinig MM, Ouverney CC, Palm K, Armitage GC, Relman DA. Methanogenic Archaea and human periodontal disease. Proc Natl Acad Sci U S A. 2004:101:6176-81.

57. Hajishengallis G, Liang S, Payne MA, Hashim A, Jotwani R, Eskan MA, et al. Low-abundance biofilm species orchestrates inflammatory periodontal disease through the commensal microbiota and complement. Cell Host Microbe. 2011:10:497-506.

58. Hajishengallis G, Darveau RP, Curtis MA. The keystone-pathogen hypothesis. Nat Rev Microbiol. 2012:10:717-25.

59. Aruni W, Chioma O, Fletcher HM. Filifactor alocis: The newly discovered kid on the block with special talents. J Dent Res. 2014:93:725-32

60. Schlafer S, Riep B, Griffen AL, Petrich A, Hübner J, Berning M, et al. Filifactor alocis - involvement in periodontal biofilms. BMC Microbiol. 2010;10:66.

61. Haffajee AD, Teles RP, Socransky SS. Association of Eubacterium nodatum and Treponema denticola with human periodontitis lesions. Oral Microbiol Immunol. 2006:21:269-82.

62. Pöllänen MT, Paino A, Ihalin R. Environmental stimuli shape biofilm formation and the virulence of periodontal pathogens. Int J Mol Sci. 2013;14:17221-37.

63. Fives-Taylor PM, Meyer DH, Mintz KP, Brissette C. Virulence factors of Actinobacillus actinomycetemcomitans. Periodontol 2000. 1999;20:136-67.

64. Jain S, Darveau RP. Contribution of Porphyromonas gingivalis lipopolysaccharide to periodontitis. Periodontol 2000. 2010;54:53-70.

65. Murray DA, Wilton JMA. Lipopolysaccharide from the periodontal pathogen Porphyromonas gingivalis prevents apoptosis of HL60-derived neutrophils in vitro. Infect Immun. 2003;71:7232-5.

66. Hiroi M, Shimojima T, Kashimata M, Miyata T, Takano H, Takahama M, et al. Inhibition by Porphyromonas gingivalis LPS of apoptosis induction in human peripheral blood polymorphonuclear leukocytes. Anticancer Res. 1998;18:3475-9.

67. Preshaw PM, Schifferle RE, Walters JD. Porphyromonas gingivalis lipopolysaccharide delays human polymorphonuclear leukocyte apoptosis in vitro. J Periodontal Res. 1999;34:197-202.

68. Tang-Larsen J, Claesson R, Edlund MB, Carlsson J. Competition for peptides and amino acids among periodontal bacteria. J Periodontal Res. 1995;30:390-5

69. Yoshida A, Yoshimura M, Ohara N, Yoshimura S, Nagashima S, Takehara T, et al. Hydrogen sulfide production from cysteine and homocysteine by periodontal and oral bacteria. J Periodontol. 2009;80:1845-51.

70. Gries CM, Bose JL, Nuxoll AS, Fey PD, Bayles KW. The Ktr potassium transport system in Staphylococcus aureus and its role in cell physiology, antimicrobial resistance and pathogenesis. Mol Microbiol. 2013;89:760-73.

71. Liu Y, Ho KK, Su J, Gong H, Chang AC, Lu S. Potassium transport of Salmonella is important for type III secretion and pathogenesis. Microbiology. 2013;159:1705-19.

72. Front E, Laster Z, Unis R, Gavish M, Nagler RM. Salivary biomarker analysis complementing regular clinical examination. Biomark Med. 2013;7:701-8.

73. Koregol AC, More SP, Nainegali S, Kalburgi N, Verma S. Analysis of inorganic ions in gingival crevicular fluid as indicators of periodontal disease activity: A clinico-biochemical study. Contemp Clin Dent. 2011:2:278-82.

74. Wyckoff EE, Boulette ML, Payne SM. Genetics and environmental regulation of Shigella iron transport systems. Biometals. 2009;22:43-51.

75. Llamas MA, Imperi F, Visca P, Lamont IL. Cell-surface signaling in Pseudomonas: stress responses, iron transport, and pathogenicity. FEMS Microbiol Rev. 2014;38:569-97.

76. Heuston S, Begley M, Gahan CGM, Hill C. Isoprenoid biosynthesis in bacterial pathogens. Microbiology. 2012;158:1389-401. 
77. Ostrovsky D, Diomina G, Lysak E, Matveeva E, Ogrel O, Trutko S. Effect of oxidative stress on the biosynthesis of 2-C-methyl-D-erythritol-2,4-cyclopyrophosphate and isoprenoids by several bacterial strains. Arch Microbiol. 1998;171:69-72.

78. Gill SR, Pop M, Deboy RT, Eckburg PB, Turnbaugh PJ, Samuel BS, et al. Metagenomic analysis of the human distal gut microbiome. Science. 2006;312:1355-9.

79. Heinzerling HF, Olivares M, Burne RA. Genetic and transcriptional analysis of flgB flagellar operon constituents in the oral spirochete Treponema denticola and their heterologous expression in enteric bacteria. Infect Immun. 1997;65:2041-51.

80. Lux R, Miller JN, Park NH, Shi W. Motility and chemotaxis in tissue penetration of oral epithelial cell layers by Treponema denticola. Infect Immun. 2001;69:6276-83.

81. Van Winkelhoff AJ, Winkel EG, Barendregt D, Dellemijn-Kippuw N, Stijne A, van der Velden U. beta-Lactamase producing bacteria in adult periodontitis. J Clin Periodontol. 1997;24:538-43.

82. Brook I. The role of beta-lactamase-producing-bacteria in mixed infections. BMC Infect Dis. 2009;9:202.

83. Barrangou R, Fremaux C, Deveau H, Richards M, Boyaval P, Moineau S, et al. CRISPR Provides Acquired Resistance Against Viruses in Prokaryotes. Science. 2007;315:1709-12.

84. Louwen R, Staals RHJ, Endtz HP, van Baarlen P, van der Oost J. The role of CRISPR-Cas systems in virulence of pathogenic bacteria. Microbiol Mol Biol Rev. 2014;78:74-88

85. Endo A, Watanabe T, Ogata N, Nozawa T, Aikawa C, Arakawa S, et al Comparative genome analysis and identification of competitive and cooperative interactions in a polymicrobial disease. ISME J. 2015;9:629-42.

86. Naito M, Sato K, Shoji M, Yukitake H, Ogura Y, Hayashi T, et al. Characterization of the Porphyromonas gingivalis conjugative transposon CTnPg1: determination of the integration site and the genes essential for conjugal transfer. Microbiology. 2011;157:2022-32.

87. Tribble GD, Garza JJ, Yeung VL, Rigney TW, Dao D-HV, Rodrigues PH, et al. Genetic analysis of mobile tetQ elements in oral Prevotella species. Anaerobe. 2010;16:604-9.

88. Nikolich MP, Hong G, Shoemaker NB, Salyers AA. Evidence for natural horizontal transfer of tetQ between bacteria that normally colonize humans and bacteria that normally colonize livestock. Appl Environ Microbiol. 1994;60:3255-60.

89. Arzese AR, Tomasetig L, Botta GA. Detection of tetQ and ermF antibiotic resistance genes in Prevotella and Porphyromonas isolates from clinical specimens and resident microbiota of humans. J Antimicrob Chemother. 2000;45:577-82.

90. Berezow AB, Darveau RP. Microbial shift and periodontitis. Periodontol 2000. 2011;55:36-47.

91. Relman DA. The human microbiome: ecosystem resilience and health. Nutr Rev. 2012;70:S2-9.

92. López R, Dahlén G, Retamales C, Baelum V. Clustering of subgingival microbial species in adolescents with' periodontitis. Eur J Oral Sci. 2011;119:141-50.

93. Mulcahy LR, Burns JL, Lory S, Lewis K. Emergence of Pseudomonas aeruginosa strains producing high levels of persister cells in patients with cystic fibrosis. J Bacteriol. 2010;192:6191-9.

94. Ciofu O, Hansen CR, Høiby N. Respiratory bacterial infections in cystic fibrosis. Curr Opin Pulm Med. 2013;19:251-8

95. Armitage GC. Analysis of gingival crevice fluid and risk of progression of periodontitis. Periodontol 2000. 2004;34:109-19.

96. Kadowaki T, Nakayama K, Okamoto K, Abe N, Baba A, Shi Y, et al. Porphyromonas gingivalis proteinases as virulence determinants in progression of periodontal diseases. J Biochem (Tokyo). 2000;128:153-9.

97. Roper JM, Raux E, Brindley AA, Schubert HL, Gharbia SE, Shah HN, et al. The enigma of cobalamin (Vitamin B12) biosynthesis in Porphyromonas gingivalis identification and characterization of a functional corrin pathway. J Biol Chem. 2000;275:40316-23.

98. Datasets used in these analyses was deposited at the Human Oral Microbiome Database (HOMD) under the submission number 20141024 [ftp://ftp.homd.org/publication_data/20141024/]

99. Datasets used in these analyses was deposited at the Human Oral Microbiome Database (HOMD) under the submission number 20130522 [ftp://ftp.homd.org/publication_data/20130522/]

\section{Submit your next manuscript to BioMed Central and take full advantage of:}

- Convenient online submission

- Thorough peer review

- No space constraints or color figure charges

- Immediate publication on acceptance

- Inclusion in PubMed, CAS, Scopus and Google Scholar

- Research which is freely available for redistribution

Submit your manuscript at www.biomedcentral.com/submit 